\title{
A Malignant Omental Extra-Gastrointestinal Stromal Tumor: Case Report and Review of the Literature
}

\author{
F. Haddar $^{1 *}$, F. Bellouhou ${ }^{1}$, A. Ait Errami ${ }^{1}$, S. Oubaha ${ }^{2}$, K. Krati ${ }^{1}$, Z. Samlani ${ }^{1}$
}

${ }^{1}$ Department of Gastroenterology, Mohammed VI University Hospital, Marrakech, Morocco

${ }^{2}$ Department of Physiology, Faculty of Medicine and Pharmacy at Cadi Ayyad University, Marrakech, Morocco

DOI: $10.36347 /$ simcr.2020.v08i02.016

| Received: 20.01.2020 | Accepted: 27.01.2020 | Published: 14.02.2020

*Corresponding author: F. Haddar

Abstract

Extra gastrointestinal stromal tumours (EGIST) are rare neoplasms of the abdomen which have similar morphological and immunohistological features of gastrointestinal stromal tumours. These tumours are not connected to either stomach or intestine and most commonly arise from mesentery, retroperitoneum, omentum etc. We describe a case of EGIST arising from omentum in a 65 year old female patient who presented with abdominal pain.

Keywords: EGIST, Omentum, CT, immunohistochemistry, imatinib, prognosis.

Copyright @ 2020: This is an open-access article distributed under the terms of the Creative Commons Attribution license which permits unrestricted use, distribution, and reproduction in any medium for non-commercial use (NonCommercial, or CC-BY-NC) provided the original author and source are credited.

\section{INTRODUCTION}

Gastrointestinal stromal tumors (GISTs) are rare neoplasms that represent only $0.1-3 \%$ of all gastrointestinal malignancies. However, they are the most common mesenchymal neoplasms of the digestive tract, accounting for $80 \%$ of all cases [1]. Its incidence has increased considerably in the past years due to growing investigation and knowledge on the features of this disease; in fact, many of the previously reported mesenchymal gastrointestinal tumors are now thought to have been misdiagnosed.

Extragastrointestinal stromal tumors (EGISTs) are a rare subgroup that display no connection to the walls of the gastrointestinal tubular organs. They are usually located in the omentum or in the mesentery and account for 5\%-10\% of all GISTs [2] Even though they share some morphological features with GISTs, the diagnosis is hard and may be made through CT-guided puncture and immunohistochemical analysis of the biopsy [3]. There are three histological types: spindle $(70 \%)$, epithelial $(20 \%)$, and mixed-cell. In $95 \%$, there is somatic mutation of CD117 (c-kit), and its discovery in the immunohistochemical characteristic defines the GIST [4]. Staging could be done with abdominal and pelvis tomography, MRI, or PET-CT [5].

\section{CaSe Report}

65-year-old female patient with no significant pathological history had consulted for epigastralgies of progressive installation, without transit disorders or fever or gastrointestinal bleeding. The clinical examination found a palpable mass at the level of the epigastrium $7 \mathrm{~cm}$ long axis of irregular contours, painless with firm consistency. Oesogastroduodenal fibroscopy had shown an aspect of extrinsic compression of the posterior side of the stomach without involvement of the wall. A Abdominal computed tomography (Figure-1) showed a mass of the epiploon cavity, measuring $74 \times 50 \mathrm{~mm}$, of compartmentalized liquid density with a marginal contingent taking the contrast measuring about $20 \mathrm{~mm}$, the mass drives back the stomach and comes into contact with the left liver and pancreas with respect for the cleavage planes.

The patient was operated; intraoperative exploration had revealed a tumor in the posterior cavity of the omentum. The stomach, left liver and pancreas were strictly normal, in particular without any tumor lesions. The patient had a complete tumor resection. The postoperative follow-ups were simple.

At the cut, the tumor was solido-cystic nodular, not encapsulated measuring $5 \times 5 \times 4.5 \mathrm{~cm}$, the cystic part had a thin multilocular wall the content was greenish red. The solid part was whitish with a firm consistency.

Microscopically, the neoplasm was surrounded by epiploic adipose tissue and all resected margins were free of the tumour. Histological examination (Figure-2), revealed a proliferation of round vacuolated epithelioid 
fusocellular cells arranged in long tangled bundles with a mitotic count estimated at 20 mitoses per field at high magnification. The nuclei were oval with a fine vesicular chromafine, discreetly anisokaryotic. They were the seat of some mitotic figures. The cytoplasm was abundant eosinophilic with the presence of juxtanuclear vacuoles. The stroma was small, fibrous and had mono-nucleated inflammatory elements and a few thin-walled blood vessels but necrosis was absent. Presence of cystic changes. The limits of resection passed into healthy tissue. The immunohistochemical study showed an extremely positive immunostaining of tumor cells for CD117, Dog and nuclear expression of $10 \%$ of Ki67 tumor cells.

The adjuvant therapy with imatinib mesylate was not performed because the tumor was totally excised (resection R0) and it was classified as a moderate risk form. No adjuvant therapy with imatinib has been prescribed. The patient is now on close followup and after six months from diagnosis is free from any local recurrence or distant metastasis. A control scanner made at 3 and 6 months evolution shows the absence of tumor recurrence.
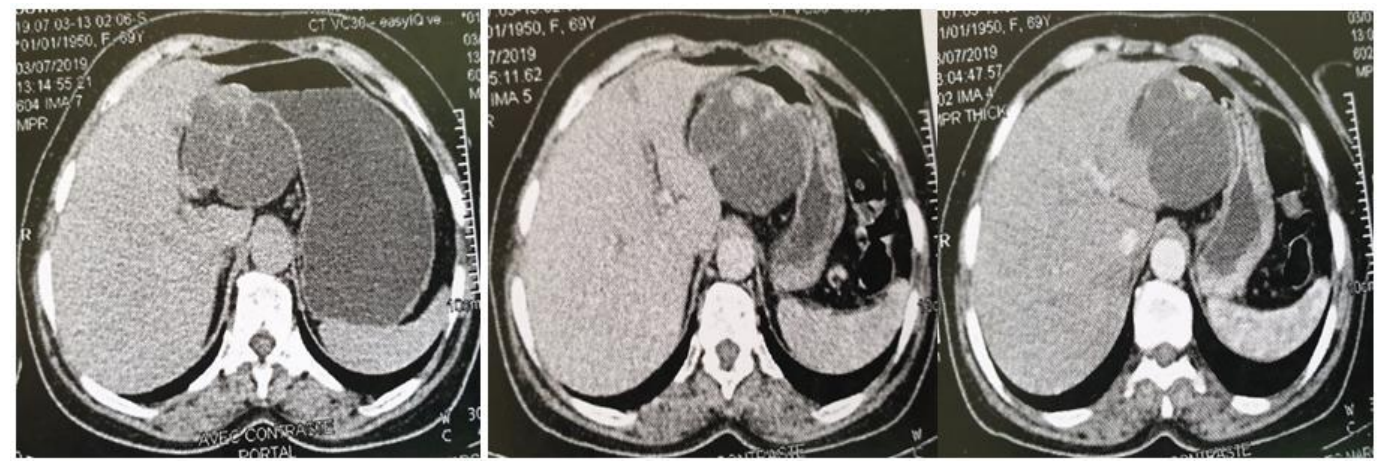

Fig-1: CT images at different levels demonstrates a large $5 \times 7,4 \mathrm{~cm}$ intraabdominal mass
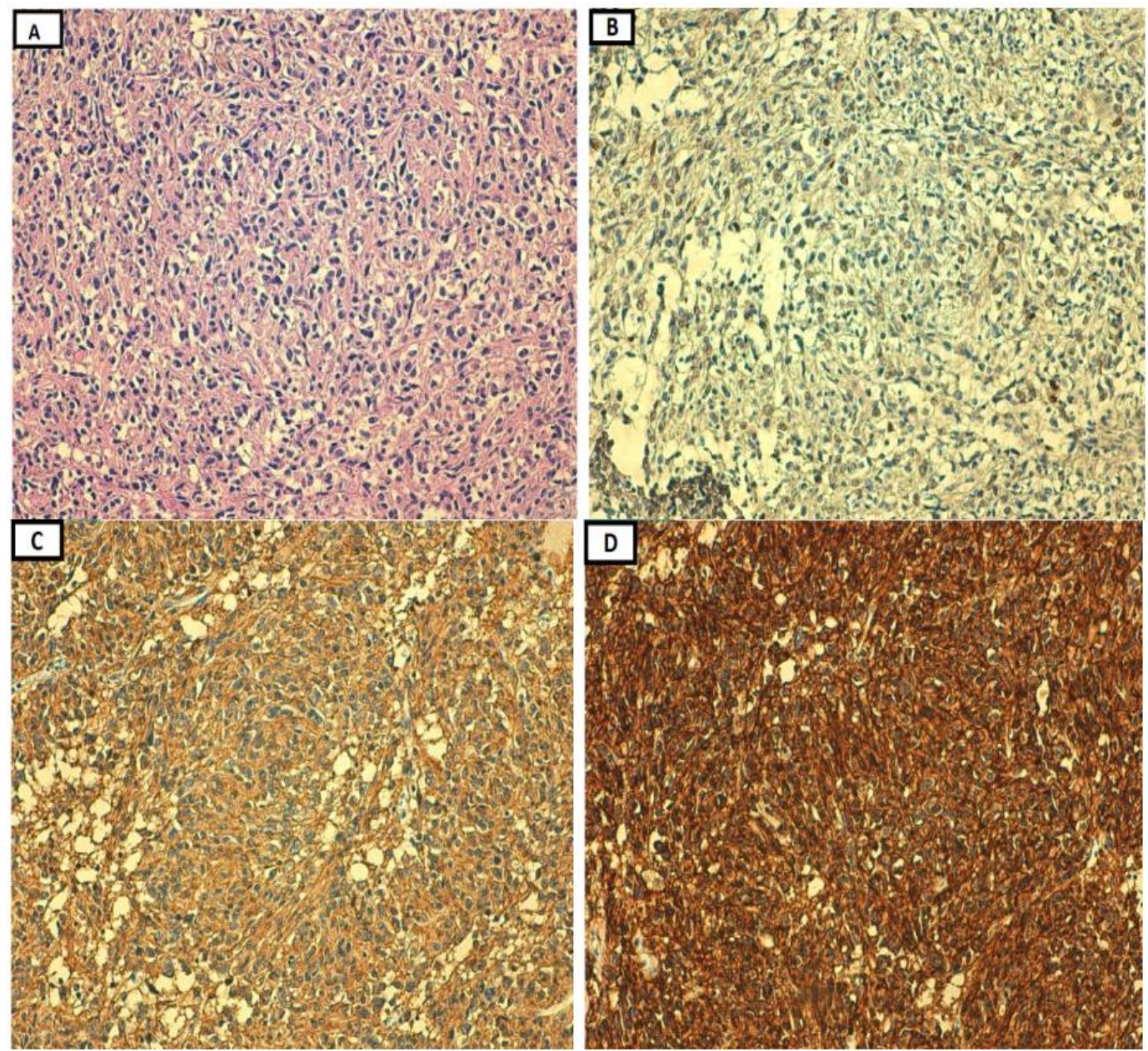

Fig-2: A: Microscopic imaging of the tumor at 20×, shown with H\&E staining B: immunohistochemistry of Ki-67 20× C: IHC Cytoplasmic expression of CD117 20×. D: IHC DOG-1 20× 


\section{DISCUSSION}

The term GIST was initially proposed by Mazur and Clark in 1983 to define intra-abdominal nonepithelial neoplasms that lacked the ultrastructural features of smooth muscle or nerve cells $[6,7]$. Today, the imunohistochemical profile is essential in the diagnosis of this tumor, with an incidence rate that nearly doubled within the last years [6]. In fact, many GISTs were previously misclassified as other gastrointestinal mesenchymal neoplasms, making the true incidence of this tumor difficult to assess [6].

In contrast to GISTs, the histogenesis of EGISTs is still poorly understood. Unlike stromal tumors of the digestive tract (GIST), that originates from the interstitial cells of Cajal (ICC), which are pacemaker cells present in the myenteric (Auerbach) plexus of the enteric nervous system of the gastrointestinal tract [8]. EGIST originates from ICClikes cells. The tumor expression of CD117 is almost constant and constitutes the criterion diagnosis cells, tumor cells show similar patterns of mutation of c-Kit and PDGFRA genes to GIST [9]. The majority of GISTs are sporadic but some may be part of hereditary disorders such as Von Recklinghausen's neurofibromatosis [10], Carney's triad, and CarneyStratakis syndrome [1]

Clinically these extragastrointestinal stromal tumors may remain asymptomatic for a long time because of their deep localization and extraluminal development, however they may manifest as vague abdominal pain or the discovery of an abdominal mass as it is the case in our observation [11]. Endoscopy and biopsy are usually not diagnostic if the mucosa is intact [1].

Preoperative diagnosis of EGIST can be done to medical imaging (CT or MRI) that allows the visualization of the tumor and can guide a fine-needle biopsy puncture for diagnostic purposes. According to Ortiz-Reyetal [12], this simple gesture is very useful and widely indicated in the EGIST. On the other hand, the exact origin of tumor proliferation is not always easily determined by imaging [13]. In our observation, the scanner showed a mass of the epiploon cavity, measuring $74 * 50 \mathrm{~mm}$, of compartmentalized liquid density with a marginal contingent taking the contrast measuring about $20 \mathrm{~mm}$, the mass drives back the stomach and comes into contact with the left liver and pancreas with respect for the cleavage planes. By getting based on the literature data, it appears that the balance systematic extension of an abdominal mass of nature imprecise must include an ultrasound and a CT scan abdominal, the other exams to be discussed on a case by case basis $[13,14]$.

The definitive diagnosis of GIST is made with the morphology which reveals a mesenchymal tumor with imunohistochemistry positive for CD-117 and
DOG1, and negative for desmin and S100 [8]. Histologically, there are three types of GISTs: spindle celled (70\%), epithelioid (20\%) and mixed [9]. Approximately $95 \%$ of GISTs carry an activating somatic mutation of CD117 [15]. CD117 is the product of proto-onco-gene c-KIT, a tyrosine kinase transmembrane receptor located on chromosome 4 (4q11-q12) [16]. Positive immunohistochemical staining for CD117 is a defining feature of GISTs [4]; however there are GISTs that have a mutation of PDGFRA instead of c-kit and therefore they do not show the characteristic CD117 positive immunostaining. C-kit positive tumors are most responsive to the treatment with c-kit selective tyrosine kinase inhibitor, STI-571; some reports support a therapeutic trial of STI-571 for all GIST patients regardless of CD117 expression [4]. Yamamoto et al., suggests that the application of STI-571 could be a therapeutic strategy for EGISTs since they have kit alterations [17]. Todoroki et al., used STI-571 (300 $\mathrm{mg} /$ day orally) as adjuvant postoperative treatment in a 65-year-old female with a primary omental stromal tumor after R0 resection with a disease free follow-up at six months [18]. The American College of Surgeons Committee on Cancer (ACOSOG) tested the benefit of adjuvant STI-571 with $400 \mathrm{mg} /$ day for one year in patients after complete resection of high-risk tumors primary GISTs. The risk of recurrence after resection of a primary GIST is high. Conventional chemotherapy has proven ineffective against GIST (less than 10\% response). The use of adjuvant STI-571 is based on the assumption of highest impact on residual microscopic disease, despite a negative margin of resection of the primary tumor [19]. STI-571 has demonstrated favorable response in more than half of patients with advanced and unresectable or metastatic GIST [20]. There has been reported resistance to STI-571 in patients with metastatic or recurrent disease, to which there are no good therapeutics currently [21].

Prognostic factors are the mitotic rate, tumour size and tumour site (gastric GISTs have a better prognosis than small bowel or rectal GISTs). Tumour rupture is an additional adverse prognostic factor and should be recorded, regardless of whether it took place before or during surgery. Mutational status has not been incorporated in any risk classification at present, although some genotypes have a distinct natural history and, above all, KIT/PDGFRA WT GISTs have peculiar clinical presentations and course. Localised GIST with PDGFR D842V mutation are generally associated with a good prognosis and resistance to imatinib. Several risk classifications have been proposed. A widely used risk classification was proposed by the Armed Forces Institute of Pathology, which incorporates the primary mitotic count, tumour size and tumour site, i.e. the three main prognostic factors in localised GISTs [22, 23]. Stratified survival analysis showed that EGISTs that originated from the omentum had the best prognosis and that those from the retroperitoneum had the worst 
F. Haddar et al., Sch J Med Case Rep, Feb., 2020; 8(2): 165-169

prognosis. After reviewing the previous literature, we believe that the above observation may be due to the following reasons. First, this observation could be due to the biological characteristics of EGISTs in the lesser omental sac being similar to those of gastric stromal tumors, and the morphological and biological characteristics of mesenteric stromal tumors being similar to those of small intestinal stromal tumors; therefore, GISTs with the origin in the mesentery have a poor prognosis [24]. Second, it could be related to the thoroughness of surgical treatment. The omentum is a free intraperitoneal organ, which facilitates complete tumor resection, whereas the distribution of blood vessels and nerves inherent to the mesentery can affect complete resection of the tumor $[25,26]$.

\section{Conclusion}

EGISTs are a little known disease entity, which may represent a potential diagnostic pitfall. The clinical symptoms of EGISTs are often manifested as abdominal pain/discomfort. Because it usually does not affect the gastrointestinal tract, an EGIST rarely causes gastrointestinal bleeding, obstruction and other typical clinical manifestations. Survival analysis showed that the primary tumor site, tumor size, and tumor cell nuclear pleomorphism are important factors affecting the prognosis of EGIST patients. While answers to the use of STI-571 in an adjuvant or even neoadjuvant setting are found, the management of patients with GIST or EGIST tumors at high risk of recurrence, such as ours, will be based on the clinical judgment of the treating physician and the availability of clinical trials.

\section{REFERENCES}

1. Sousa D, Allen M, Mateus A, Cruz A, Marinho D, Ferreira A, Cusati P, Martins JA. EGIST: a rare tumor. Journal of gastrointestinal cancer. 2018 Jun 1;49(2):182-5.

2. Fagkrezos D, Touloumis Z, Giannila M, Penlidis C, Papaparaskeva K, Triantopoulou C. Extragastrointestinal stromal tumor of the omentum: a rare case report and review of the literature. Rare Tumors. 2012; 4(3):e44.

3. Trombatore C, Palmucci S, Angelico G, Vasquez E, Petrillo G, Puleo S, Di Cataldo A. Extragastrointestinal stromal tumor of lesser omentum: a challenging radiological and histological diagnosis. Clinical imaging. $2015 \mathrm{Nov}$ 1;39(6):1123-7.

4. Dorfman DM, Bui MM, Tubbs RR, Hsi ED, Fitzgibbons PL, Linden MD, Rickert RR, Roche PC. The CD117 immunohistochemistry tissue microarray survey for quality assurance and interlaboratory comparison: a College of American Pathologists Cell Markers Committee Study. Archives of pathology \& laboratory medicine. 2006 Jun;130(6):779-82.

5. National Comprehensive Cancer Network. Soft tissue sarcoma, version 2.2016, NCCN clinical practice guidelines in oncology. http://www.jnccn.org/content/14/6/758.full.

6. Raut C, Pawlik T. Gastrointestinal stromal tumors. In: Current surgical therapy. 11th ed: ElsevierSaunders, 2014; 96-103.

7. Feldman M. Gastrointestinal stromal tumors (GISTs). In: Sleisenger and Fordtran's gastrointestinal and liver disease. 10th ed: Elsevier- Saunders; 2016, 487-500.

8. Rubin B. GIST and EGIST. In: Enzinger and Weiss's soft tissue tumors. 6th ed: ElsevierSaunders; 2014, 569-90.

9. Fletcher CD, Berman JJ, Corless C, Gorstein F, Lasota J, Longley BJ, Miettinen M, O'Leary TJ, Remotti H, Rubin BP, Shmookler B. Diagnosis of gastrointestinal stromal tumors: a consensus approach. International journal of surgical pathology. 2002 Apr;10(2):81-9.

10. Miettinen M, Fetsch JF, Sobin LH, Lasota J. Gastrointestinal stromal tumors in patients with neurofibromatosis 1: a clinicopathologic and molecular genetic study of 45 cases. The American journal of surgical pathology. 2006 Jan 1;30(1):90-6.

11. Pandey R, Kochar R. Management of gastrointestinal stromal tumors: looking beyond the knife. An update on the role of adjuvant and neoadjuvant imatinib therapy. Journal of gastrointestinal cancer. 2012 Dec 1;43(4):547-52.

12. Ortiz-Rey JA, Fernández GC, Magdalena CJ, Álvarez C, Antón I, San Miguel P, De la Fuente A. Fine needle aspiration appearance of extragastrointestinal stromal tumor. Acta cytologica. 2003;47(3):490-4.

13. Cananzi FC, Judson I, Lorenzi B, Benson C, Mudan S. Multidisciplinary care of gastrointestinal stromal tumour: a review and a proposal for a pretreatment classification. European Journal of Surgical Oncology (EJSO). 2013 Nov 1;39(11):1171-8.

14. Bouassria A, Mouaqit O, Benjelloun E, Elbouhaddouti H, Ousadden A, Mazaz K, Taleb KA. Exérèse curative d'une tumeur stromale extra gastro intestinale (EGIST) localement avancée et fistulisée dans le grêle après traitement par Imatinib: à propos d'un cas. The Pan African Medical Journal. 2014;17.

15. Agaimy A, Wünsch PH. Gastrointestinal stromal tumours: a regular origin in the muscularis propria, but an extremely diverse gross presentation. Langenbeck's archives of surgery. 2006 Aug 1;391(4):322-9.

16. Blackstein ME, Rankin B, Fletcher C, Heinrich M, Benjamin R, Mehren MV, Blanke C, Fletcher JA, Borden E, Demetri G. Clinical benefit of Imatinib in patients with metastatic GIST negative for the expression of CD117 in the S0033 trial. ASCO. J Clin Oncol. 2005;9010.

17. Yamamoto H, Oda Y, Kawaguchi K, Nakamura N, Takahira T, Tamiya S, Saito T, Oshiro Y, Ohta M, 
Yao T, Tsuneyoshi M. c-kit and PDGFRA mutations in extragastrointestinal stromal tumor (gastrointestinal stromal tumor of the soft tissue). The American journal of surgical pathology. 2004 Apr 1;28(4):479-488.

18. Todoroki T, Sano T, Sakurai S, Segawa A, Saitoh T, Fujikawa K, Yamada S, Hirahara N, Tsushima Y, Motojima R, Motojima T. Primary omental gastrointestinal stromal tumor (GIST). World journal of surgical oncology. 2007 Dec;5(1):66.

19. Castillo-Sang M, Mancho S, Tsang AW, Gociman B, Almaroof B, Ahmed MY. A malignant omental extra-gastrointestinal stromal tumor on a young man: a case report and review of the literature. World journal of surgical oncology. 2008 Dec;6(1):50.

20. Demetri GD, Von Mehren M, Blanke CD, Van den Abbeele AD, Eisenberg B, Roberts PJ, Heinrich MC, Tuveson DA, Singer S, Janicek M, Fletcher JA. Efficacy and safety of imatinib mesylate in advanced gastrointestinal stromal tumors. New England Journal of Medicine. 2002 Aug 15;347(7):472-80.
21. Dematteo RP, Heinrich MC, Wa'el M ER, Demetri G. Clinical management of gastrointestinal stromal tumors: before and after STI-571. Human pathology. 2002 May 1;33(5):466-77.

22. Miettinen M, Lasota J. Gastrointestinal stromal tumors: review on morphology, molecular pathology, prognosis, and differential diagnosis. Archives of pathology \& laboratory medicine. 2006 Oct;130(10):1466-78.

23. Miettinen M, Lasota J. Gastrointestinal stromal tumors: pathology and prognosis at different sites. InSeminars in diagnostic pathology 2006 May 1; 23(2):70-83.

24. Shetty N, Sirohi B, Shrikhande SV. Molecular target therapy for gastrointestinal stromal tumors. Gan To Kagaku Ryoho 2015; 4: 207-218.

25. Trinh VA, You Y, Hwu WJ. Treatment of BRAFmutated advanced cutaneous melanoma. Chinese clinical oncology. 2014 Mar 6;3(3):28.

26. Zhou J, Yan T, Huang Z, Zhao J, Bi X, Zhao H, Zhang Y, Cai J. Clinical features and prognosis of extragastrointestinal stromal tumors. Int J Clin Exp Med. 2016 Jan 1;9(8):16367-72. 